\title{
T Wave Notched by ECG Finding
}

National Cancer Institute

\section{Source}

National Cancer Institute. I Wave Notched by ECG Finding. NCI Thesaurus. Code C71032.

An electrocardiog raphic finding of an irregular, u or v shaped deflection within the contour of the T wave. (CDISC) 